\title{
Testing gravity using dwarf stars
}

\author{
Jeremy Sakstein \\ Institute of Cosmology and Gravitation, University of Portsmouth, Portsmouth PO1 3FX, United Kingdom
}

(Received 30 October 2015; published 21 December 2015)

\begin{abstract}
Generic scalar-tensor theories of gravity predict deviations from Newtonian physics inside astrophysical bodies. In this paper, we point out that low mass stellar objects, red and brown dwarf stars, are excellent probes of these theories. We calculate two important and potentially observable quantities: the radius of brown dwarfs and the minimum mass for hydrogen burning in red dwarfs. The brown dwarf radius can differ significantly from the general relativity prediction, and upcoming surveys that probe the mass-radius relation for stars with masses $<\mathcal{O}\left(0.1 M_{\odot}\right)$ have the potential to place new constraints. The minimum mass for hydrogen burning can be larger than several presently observed red dwarf stars. This places a new and extremely stringent constraint on the parameters that appear in the effective field theory of dark energy and rules out several well-studied dark energy models.
\end{abstract}

DOI: 10.1103/PhysRevD.92.124045

PACS numbers: 04.50.Kd, 95.36.+x, 97.20.Vs, 98.80.-k

\section{INTRODUCTION}

Dark energy and the cosmological constant problem have been driving the study of alternative theories of gravity for more than a decade (see [1,2] for recent reviews). Theories that are able to drive the acceleration of the cosmic expansion typically invoke screening mechanisms in order to remain compatible with local tests of gravity. These use nonlinear features to decouple solar system and cosmological scales such that the theory behaves like general relativity (GR) in the solar system while exhibiting novel cosmologies. This has prompted a large theoretical effort toward finding novel small scale probes of these mechanisms, and astrophysical probes, in particular stellar structure tests, have emerged as promising candidates [3-11].

The Vainshtein mechanism [12] is very efficient at hiding deviations from GR locally and is exhibited in a wide variety of alternative gravity theories [13-15]. Recently, the authors of [16] have shown that the mechanism is partially broken inside astrophysical bodies in a wide class of interesting theories (the beyond Horndeski class $[17,18]$ ). Using this result, the authors of [11] have derived the modifications to the stellar structure equations, ${ }^{1}$ which manifest as a modification of the hydrostatic equilibrium equation. This was applied to polytropic models to derive the new Lane-Emden equation, which was used to predict the properties of main-sequence stars. The breaking of the mechanism can result in weaker gravity inside extended objects, and this results in stars that are dimmer and cooler at fixed mass. ${ }^{2}$ Polytropic models are an incomplete model of main-sequence stars and lack a description of nuclear burning and metallicity effects. As such, the authors of

\footnotetext{
jeremy.sakstein@port.ac.uk

${ }^{1}$ See also [19].

${ }^{2}$ There is a small region of parameter space where the converse is true [19]. We will discuss this later.
}

[11] identified several degeneracies that may make the predicted novel effects difficult to observe in practice.

In this paper, we point out that low mass stellar objects, red and brown dwarf stars, are perfect probes of these theories precisely because they are well described by polytropic models. They are chemically homogeneous except for the photosphere, and their observational properties are only weak functions of their opacity. Furthermore, the lack of chemical evolution (except during their very early phases) allows us to treat them as static.

Low mass brown dwarfs have a radius that is almost independent of their mass over a large range of masses. Polytropic models based on GR predict that this is $0.1 R_{\odot}$, which is close to the observed value. In this work, we find that this can be arbitrarily large when gravity is weaker, and as small as $0.039 R_{\odot}$ when it is stronger. Measuring the massradius relation of objects with mass $M \lesssim \mathcal{O}\left(0.1 M_{\odot}\right)$ can therefore place a new constraint on alternative gravity theories.

Higher mass objects obey a different mass-radius relation whereby they are more compact at higher masses. Objects that are massive enough have sufficiently high core temperatures and densities to burn hydrogen. Thus, only stars that are sufficiently massive can burn on the main sequence; lower mass objects remain inert and eventually cool. GR predicts that the minimum mass for hydrogen burning (MMHB) is around $0.08 M_{\odot}$, which is again very close to what is observed. Here, we find that alternative gravity models predict values that can be arbitrarily large when gravity is weaker. ${ }^{3}$ The observation of several hydrogen burning stars with masses in the range $0.08-0.1 M_{\odot}$ then allows us to constrain the new parameter $\Upsilon \lesssim 0.027$. In terms of the effective field theory of dark energy, this places an independent constraint on the parameters

\footnotetext{
${ }^{3}$ We will not consider the case where gravity is stronger because this is always consistent with observations of the lowest mass hydrogen burning objects.
} 


$$
\frac{\alpha_{H}^{2}}{\alpha_{H}-\alpha_{T}-\alpha_{B}\left(1+\alpha_{T}\right)} \lesssim 0.0068 .
$$

This constraint was presented in a recent article [20]. Here, we expand the calculation and discuss it in detail.

This paper is organized as follows: In Sec. II we provide an introduction to the Vainshtein mechanism for readers unfamiliar with screening mechanisms and alternative theories of gravity. We also present the modification of the hydrostatic equilibrium equation and apply it to polytropic models to derive the new Lane-Emden equation. The connection with the effective field theory of dark energy is also presented. In Sec. III we provide a brief review of the salient features of dwarf stars for the benefit of readers well versed in alternative gravity theories who may be unfamiliar with stellar astrophysics. In Sec. IV we apply the modified Lane-Emden equation to dwarf star models in order to calculate the alternative gravity theory predictions for their observable properties. We conclude in Sec. V.

\section{ALTERNATIVE GRAVITY THEORIES AND THE VAINSHTEIN MECHANISM}

\section{A. The Vainshtein mechanism}

The Newtonian limit of GR results in a scalar theory of gravity where the gravitational field is described by the Newtonian potential $\Phi_{\mathrm{N}}$, which results in a force (per unit mass) $F_{\mathrm{N}}=-\nabla \Phi_{\mathrm{N}}$. The gravitational field is sourced by the local density $\rho$ through the Poisson equation

$$
\nabla^{2} \Phi_{\mathrm{N}}=4 \pi G \rho .
$$

Scalar-tensor alternatives to GR include an additional field $\phi$, which results in a fifth force

$$
F_{5}=-\beta \nabla \phi
$$

parametrized by a dimensionless coupling $\beta \sim \mathcal{O}(1)$. The simplest scalar-tensor theories generically predict that the local field is sourced by the density in the exact same manner as the Newtonian potential [21],

$$
\nabla^{2} \phi=8 \pi \beta G \rho,
$$

so that one has $\phi=2 \beta \Phi_{\mathrm{N}}$. The total force is then $F=\left(1+2 \beta^{2}\right) F_{\mathrm{N}}$, and one must typically tune $\beta \lesssim 10^{-3}$ to satisfy solar system bounds [21]. Importantly, one then has $\beta \ll 1$ on all scales, which makes it difficult for $\phi$ to have any nontrivial influence on the cosmology.

The Vainshtein mechanism circumvents this by introducing new derivative interactions that manifest as new differential operators on the left hand side of (4). This results in solutions for $\phi$ that differ significantly from $\Phi_{\mathrm{N}}$. One of the simplest examples of this is the cubic Galileon model [22], which, imposing spherical symmetry, results in the following equation:

$$
\frac{1}{r^{2}} \frac{\mathrm{d}}{\mathrm{d} r}\left(r^{2} \frac{\mathrm{d} \phi}{\mathrm{d} r}\right)+\frac{1}{r^{2}} \frac{\mathrm{d}}{\mathrm{d} r}\left[r\left(\frac{\mathrm{d} \phi}{\mathrm{d} r}\right)^{2}\right]=8 \pi \beta G \rho .
$$

The first term can be recognized as $\nabla^{2} \phi$; the second is the new term arising from the new interaction. Integrating once, we obtain an algebraic relation for the ratio of the fifth to Newtonian force $x \equiv F_{5} / F_{\mathrm{N}}$,

$$
x+\left(\frac{r_{\mathrm{V}}}{r}\right)^{3} \frac{x^{2}}{2 \beta^{2}}=2 \beta^{2}, \quad \text { where } r_{\mathrm{V}}^{3} \equiv \frac{G M}{\Lambda^{2}}
$$

is the Vainshtein radius and $\Lambda$ is a new mass scale that appears in the underlying theory. Far outside the Vainshtein radius, we have $x \approx 2 \beta^{2}$ and so $F_{5}=2 \beta^{2} F_{\mathrm{N}}$, the fifth force is a factor of $2 \beta^{2}$ larger than the Newtonian one. This is the unscreened regime. When $r \ll r_{\mathrm{V}}$ we instead have

$$
\frac{F_{5}}{F_{\mathrm{N}}}=2 \beta^{2}\left(\frac{r}{r_{\mathrm{V}}}\right)^{\frac{3}{2}},
$$

and the fifth force is suppressed by a factor of $\left(r / r_{\mathrm{V}}\right)^{3 / 2}$. This is the screened regime. The Vainshtein radius of the Sun is $\mathcal{O}(\mathrm{pc})$ [23], and so one does not need to tune $\beta \ll 1$ in order to satisfy solar system tests.

\section{B. Breaking of the Vainshtein mechanism and stellar structure}

The cubic Galileon is the simplest example of a theory that exhibits the Vainshtein mechanism. The authors of [16] have shown that the mechanism is partially broken inside objects of finite extent in more generic theories. Specifically, outside objects the mechanism works as above but their internal structure is governed by the modified hydrostatic equilibrium equation $[11,19]$

$$
\frac{\mathrm{d} P}{\mathrm{~d} r}=-\frac{G M \rho}{r^{2}}-\frac{\Upsilon}{4} G \rho \frac{\mathrm{d}^{2} M}{\mathrm{~d} r^{2}} .
$$

Here, $\Upsilon$ is a dimensionless parameter that characterizes the strength of the modifications of gravity; it is a combination of the new mass scales appearing in the Lagrangian for the theory and the time derivative of the cosmological field. Note that since the mass is more concentrated toward the center of stars, the new term corresponds to a weakening of gravity if $\Upsilon>0$ and a strengthening when the converse is true.

In terms of the parameters appearing in the effective field theory (EFT) of dark energy [18,24], one has ${ }^{4}$ [19]

$$
\frac{\alpha_{H}^{2}}{\alpha_{H}-\alpha_{T}-\alpha_{B}\left(1+\alpha_{T}\right)} \lesssim 0.0068 .
$$

\footnotetext{
${ }^{4}$ In general, there are two additional parameters, $\alpha_{K}$ and $M_{*}$.
} 
The EFT describes the cosmology of beyond Horndeski theories, a very general scalar-tensor theory ${ }^{5}$ where the equations of motion are second order [18], on linear scales. The parameters therefore govern deviations from GR on these scales, and upcoming surveys aimed at testing gravity will focus on them. Any independent constraint from small scales is therefore complementary to these searches. Furthermore, several viable competitors to $\Lambda \mathrm{CDM}$, for example, the covariant quartic Galileon, which has $\Upsilon=1 / 3$, predict $\Upsilon \sim \mathcal{O}(1)$ precisely because the dynamics of the theory differ greatly from GR. The constraint we will obtain from the MMHB is strong enough to rule these out. Note that $\Upsilon \propto \alpha_{H}^{2}$, which is only nonzero when the beyond Horndeski terms are present. This reflects the fact that the Vainshtein mechanism works flawlessly when the theory is pure Horndeski and so only those theories that contain some beyond Horndeski terms are probed. Since there are no symmetries protecting the general action, one would expect quantum corrections to generate such terms in generic modified gravity theories. Finally, we note that there is no upper limit on $\Upsilon$, but the authors of [19] have shown that theories where $\Upsilon<-2 / 3$ do not give stable stellar configurations. For this reason, we restrict our attention to the region $-2 / 3<\Upsilon<\infty$.

A wide variety of stellar systems can be described using a polytropic equation of state (EOS)

$$
P=K \rho^{\frac{n+1}{n}},
$$

where $K$ is a constant that depends on the composition of the fluid and $n$ is known as the polytropic index. In GR, the equations of stellar structure are scale invariant, and this allows one to reformulate them in terms of dimensionless quantities. This symmetry is preserved by Eq. (8), and so we write $r=r_{\mathrm{c}} \xi, \rho=\rho_{\mathrm{c}} \theta(\xi)^{n}$, and $P=P_{\mathrm{c}} \theta(\xi)^{n+1}$, where $P_{\mathrm{c}}$ and $\rho_{\mathrm{c}}$ are the central pressures and densities and

$$
r_{\mathrm{c}}^{2} \equiv \frac{(n+1) P_{\mathrm{c}}}{4 \pi G_{\mathrm{N}} \rho_{\mathrm{c}}^{2}}
$$

Using the continuity equation, one has

$$
\frac{\mathrm{d} M}{\mathrm{~d} r}=4 \pi r^{2} \rho(r)
$$

which implies

$$
\frac{\mathrm{d}^{2} M}{\mathrm{~d} r^{2}}=8 \pi r \rho+4 \pi r^{2} \frac{\mathrm{d} \rho}{\mathrm{d} r}
$$

Using these in conjunction with Eq. (8), one is led to the modified Lane-Emden equation [11]

\footnotetext{
${ }^{5}$ Note that the beyond Horndeski class encompasses a wide variety of healthy scalar-tensor extensions of GR, but it is by no means the most general [25-27] theory.
}

$$
\frac{1}{\xi^{2}} \frac{\mathrm{d}}{\mathrm{d} \xi}\left[\left(1+n \Upsilon \xi^{2} \theta^{n-1}\right) \xi^{2} \frac{\mathrm{d} \theta}{\mathrm{d} \xi}+\frac{\Upsilon}{2} \xi^{3} \theta^{n}\right]=-\theta^{n},
$$

which is subject to the boundary conditions $\theta(0)=1$ $\left[P(0)=P_{\mathrm{c}}, \rho(0)=\rho_{\mathrm{c}}\right]$ and $\theta^{\prime}(0)=0\left[\mathrm{~d} P / \mathrm{d} r(0)=0^{6}\right]$. The radius $R$ of the star is defined by $P(R)=0$, which defines $\xi_{R}$ such that $\theta\left(\xi_{R}\right)=0$.

In GR, $\Upsilon=0$ and (14) reduces to the Lane-Emden equation. In this case, each polytropic index $n$ has a unique solution. In alternative theories, this is no longer the case, and the solution varies with $\Upsilon$. It was shown in [11] that the solution near the origin is given by

$$
\theta(\xi)=1-\alpha \xi^{2}, \quad \text { with } \quad \alpha=\frac{1}{6}+\frac{\Upsilon}{4} .
$$

Three important dimensionless quantities are

$$
\begin{gathered}
\omega_{n} \equiv-\left.\xi^{2} \frac{\mathrm{d} \theta}{\mathrm{d} \xi}\right|_{\xi=\xi_{R}}, \\
\gamma_{n} \equiv(4 \pi)^{\frac{1}{n-3}}(n+1)^{\frac{n}{3-n}} \omega_{n}^{\frac{n-1}{3-n} \xi_{R}}, \quad \text { and } \\
\delta_{n} \equiv-\frac{\xi_{R}}{3 \mathrm{~d} \theta /\left.\mathrm{d} \xi\right|_{\xi=\xi_{R}}},
\end{gathered}
$$

which appear in the formula for the mass

$$
M=4 \pi r_{\mathrm{c}}^{3} \rho_{\mathrm{c}} \omega_{n},
$$

the mass-radius relation

$$
R=\gamma_{n}\left(\frac{K}{G}\right)^{\frac{n}{3-n}} M^{\frac{n-1}{n-3}}
$$

and the central density

$$
\rho_{\mathrm{c}}=\delta_{n}\left[\frac{3 M}{4 \pi R^{3}}\right] .
$$

\section{DWARF STARS}

In this section, we provide a brief overview of the salient features of brown dwarf theory necessary for the calculations presented later on. The reader is referred to [28] and references therein for a more detailed account.

The brown dwarf branch occupies the region between Jupiter-like gas planets $\left(M_{\mathrm{J}}=10^{-3} M_{\odot}\right)$ and mainsequence stars $\left(M \gtrsim 0.08 M_{\odot}\right)$. They are composed of molecular hydrogen and helium in the liquid metallic phase, except in a very thin layer near the surface where the density is too low and the fluid exists as a weakly

\footnotetext{
${ }^{6}$ This is a consequence of spherical symmetry.
} 
coupled plasma that satisfies the ideal gas law. They are fully convective and contract along the Hayashi track and therefore have a polytropic EOS with $n=1.5$ [29]. Highmass brown dwarfs are supported by electron degeneracy pressure, but classical Coulomb corrections shift the EOS of low mass stars $\left(M \lesssim 4 M_{\mathrm{J}}\right)$ toward $n=1$ [30,31]. Equation (20) reveals that, when this is the case, the radius is independent of the mass and is fixed by the EOS and the solution of the Lane-Emden equation alone. In GR, one finds ${ }^{7} \gamma_{1}=\sqrt{\pi / 2} \approx 1.253$ and $R \approx 0.1 R_{\odot}$. We will return to calculate this for alternative theories later in Sec. IV.

When the star is first formed, it contracts under its own self-gravity, which leads to a rise in its central temperature and density. The contraction stops at the onset of either electron degeneracy or thermonuclear fusion depending on the mass; this defines brown dwarf and red dwarf stars, respectively. Only objects that are sufficiently heavy can reach central conditions capable of thermonuclear ignition before the fluid becomes degenerate. Thus, there is a minimum mass for hydrogen burning, which is typically around $0.08 M_{\odot}$ if the theory is GR. We will calculate this mass in alternative theories in Sec. IV. If the star cannot achieve thermonuclear ignition before degeneracy sets in, it will become a brown dwarf. Degenerate gases have equations of state where the pressure is almost independent of the temperature, and so radiation from the surface does not lead to further contraction. Instead, the brown dwarf cools over time [33-35].

Typically, the central temperature and density of brown dwarfs are of order $10^{6} \mathrm{~K}$ and $10^{3} \mathrm{~g} / \mathrm{cm}^{3}$, respectively. These conditions are not sufficient to overcome the Coulomb barrier for ${ }^{3} \mathrm{He}-{ }^{3} \mathrm{He}$ and ${ }^{3} \mathrm{He}-{ }^{4} \mathrm{He}$ reactions, and so burning cannot proceed via the Proton-Proton (PP) chains. Instead, the star consumes deuterium in the following reactions:

$$
\begin{aligned}
& \mathrm{p}+\mathrm{p} \rightarrow \mathrm{d}+\mathrm{e}^{+}+\nu_{e} \\
& \mathrm{p}+\mathrm{d} \rightarrow{ }^{3} \mathrm{He}+\gamma .
\end{aligned}
$$

The second reaction is a strong interaction and therefore proceeds quickly, consuming primordial deuterium. The first is a weak process creating a new source of deuterium and is therefore the rate-limiting, bottle-neck, reaction. The energy generation rate per unit mass at brown dwarf temperatures and densities follows the approximate power-law form

$$
\epsilon_{\mathrm{pp}}=\epsilon_{\mathrm{c}}\left(\frac{T}{T_{\mathrm{c}}}\right)^{s}\left(\frac{\rho}{\rho_{\mathrm{c}}}\right)^{u-1},
$$

\footnotetext{
${ }^{7}$ Note that the Lane-Emden equation has the exact solution $\theta(\xi)=\operatorname{sinc}(\xi)$ when $\Upsilon=0$ (i.e. GR) [32], whose first zero occurs when $\xi=\pi$. Putting this into Eq. (17) gives $\gamma_{1}=\sqrt{\pi / 2}$.
}

with $s \approx 6.31$ and $u \approx 2.28 . \epsilon_{\mathrm{c}}$ is given by (see $[28,36,37]$ )

$$
\epsilon_{c}=\epsilon_{0} X^{2} T_{\mathrm{c}}{ }^{s} \rho_{\mathrm{c}}{ }^{u-1} \exp \left(\frac{0.147}{\mu_{\mathrm{e}}^{0.43}}\right)
$$

where $X$ is the hydrogen mass fraction and $\epsilon_{0}=5.2 \times 10^{-9} \mathrm{ergs} / \mathrm{g} / \mathrm{s}$.

\section{DWARF STARS IN ALTERNATIVE GRAVITY THEORIES}

This section constitutes the main original results of this paper. The derivations will closely follow the semianalytic model of Burrows and Liebert [28].

\section{A. Brown dwarfs: The radius plateau}

As remarked in Sec. III, low mass brown dwarfs $\left(M \lesssim 4 M_{\mathrm{J}}\right)$ are well modeled by $n=1$ polytropes and hence have radii given by

$$
R=\gamma_{1}\left(\frac{K}{G}\right)^{\frac{1}{2}}
$$

which is $\approx 0.1 R_{\odot}$ when $\Upsilon=0$, i.e. in GR. ${ }^{8}$ Since $K$ is independent of the theory of gravity, the radius of low mass brown dwarfs in alternative theories of gravity is given by

$$
R(\Upsilon)=0.1 \frac{\gamma_{1}(\Upsilon)}{\gamma_{1}(\Upsilon=0)} R_{\odot}
$$

This is plotted as a function of $\Upsilon$ in Fig. 1. When $\Upsilon>0$, gravity is weaker and the radius is larger than the GR prediction. When $\Upsilon<0$, gravity is stronger, and hence the radius is lower, up to the limiting value of $\Upsilon=-2 / 3$, which predicts $R=0.039 R_{\odot}$. The authors of [11] have investigated the changes in main-sequence and red giant branch stars these theories predict and have found that they are $\lesssim 1 \%$ when $\Upsilon \lesssim \mathcal{O}\left(10^{-1}\right)$. This is the first example showing that the changes to the structure of brown dwarfs can still be significant in this range and that these stars are better probes of alternative theories of gravity. Unfortunately, measurements of the mass-radius relation for low mass objects are sparse (see [38,39], Fig. 2) and so we will not attempt to constrain $\Upsilon$ using this effect. Future data releases from surveys targeted at low mass objects such as GAIA [40,41] will improve the empirically measured mass-radius relation and therefore have the potential to place new constraints on alternative gravity theories.

\footnotetext{
${ }^{8}$ The constant $K \approx 2.7 \times 10^{5} \mathrm{~m}^{5} / \mathrm{kg} / \mathrm{s}^{2}$, which is a fit to a more precise EOS [30,31].
} 


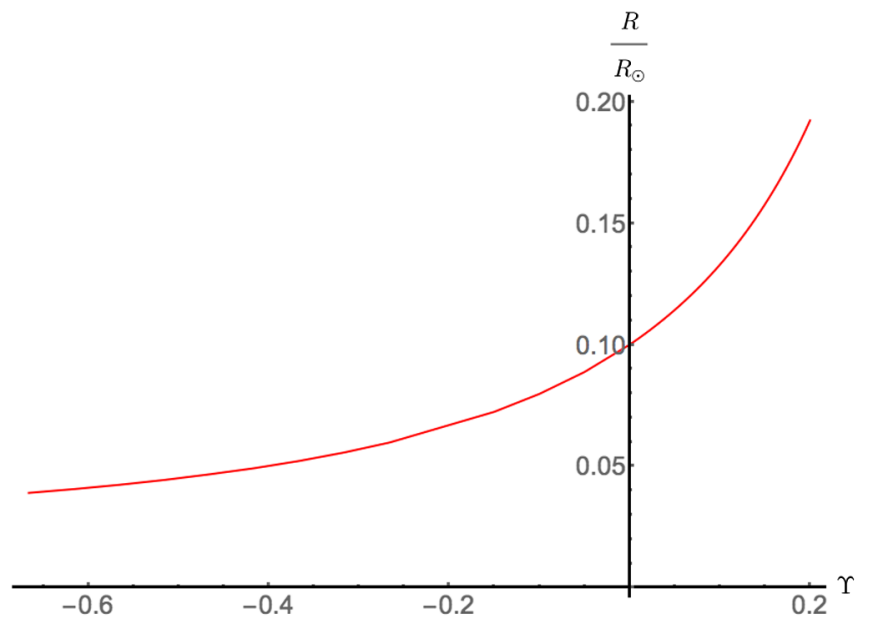

FIG. 1 (color online). The radius of low mass brown dwarf stars in alternative theories of gravity as a function of $\Upsilon$.

\section{B. High mass brown dwarfs: The minimum mass for hydrogen burning}

Higher mass brown dwarfs are supported by degeneracy pressure. A good measure of the degree of degeneracy is

$$
\eta \equiv \frac{\mu_{\mathrm{F}}}{k_{\mathrm{B}} T}=\frac{\left(3 \pi^{2}\right)^{\frac{2}{3}} \hbar^{2}}{2 m_{\mathrm{e}} m_{\mathrm{H}}^{\frac{2}{3}} k_{\mathrm{B}}} \frac{\rho^{\frac{2}{3}}}{\mu_{\mathrm{e}}^{\frac{2}{3}} T},
$$

where $\mu_{\mathrm{F}}$ is the Fermi energy, $m_{\mathrm{H}}$ is the mass of atomic hydrogen, and $\mu_{\mathrm{e}}$ is the number of baryons per electron. Note that $\rho^{1 / n} / T$ is a constant for polytropic gases [42] and so $\eta$ is a constant. For a metal poor gas with $Z=0$ (i.e. zero metallicity), which is appropriate for brown dwarfs, one has

$$
\frac{1}{\mu_{\mathrm{e}}}=X+\frac{Y}{2}=\frac{X+1}{2}
$$

with $X$ and $Y$ being the hydrogen and helium mass fractions, respectively. In this work we will not fix $\mu_{\mathrm{e}}$ in

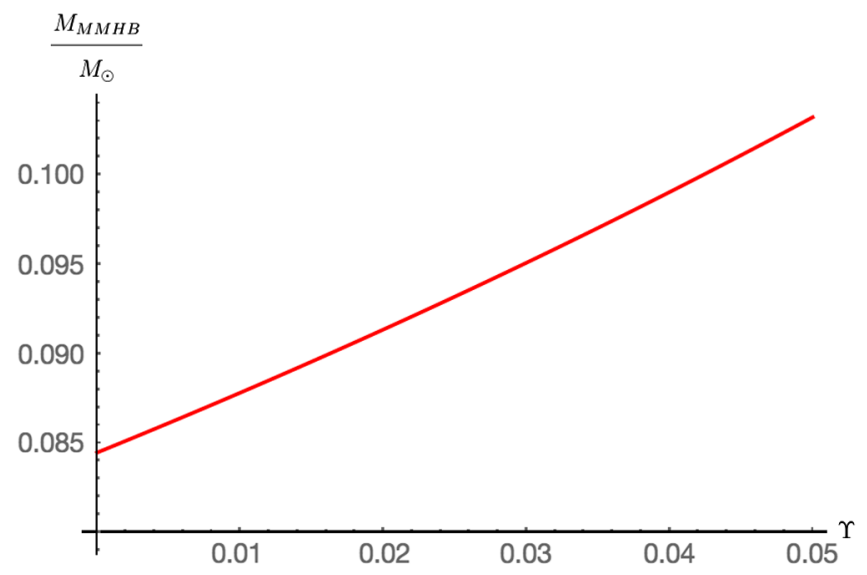

FIG. 2 (color online). The MMHB as a function of $\Upsilon$. order to show the dependency of important quantities; however, we note that for a hydrogen-helium fluid with $X=0.75, Y=0.25$, which is typical for a brown dwarf, one finds $\mu_{\mathrm{e}}=1.143$, which we will take when we compute any numerical values.

When the gas is fully degenerate, the pressure can be found by integrating over the Fermi-Dirac distribution to find

$$
P_{\mathrm{deg}}=\frac{\left(3 \pi^{2}\right)^{\frac{2}{3} \hbar^{2}}}{5 m_{\mathrm{e}} m_{\mathrm{H}}^{\frac{5}{3}}} \frac{\rho_{\mathrm{e}}^{\frac{5}{3}}}{\mu_{\mathrm{e}}^{\frac{5}{3}}} .
$$

In the other extreme, when the pressure support is due to the internal motion of the gas, the EOS is given by the idea gas law

$$
P_{\mathrm{gas}}=\frac{\rho k_{\mathrm{B}} T}{\mu m_{\mathrm{H}}}
$$

where $\mu$ is the mean molecular mass given by

$$
\frac{1}{\mu}=2 X+\frac{3 Y}{4} .
$$

Again, we will not fix the value of $\mu$ in order to show the dependency of intermediate expressions, but we note that $\mu=0.593$ for $X=0.75, Y=0.25$. We will use this value when evaluating any expressions numerically. Using Eq. (26), one has $P_{\text {gas }}=\alpha P_{\text {deg }} / \eta$, with

$$
\alpha \equiv \frac{5 \mu_{\mathrm{e}}}{2 \mu} \approx 4.82 .
$$

In general, a good approximation for the EOS between the two regimes is

$$
P=\frac{\left(3 \pi^{2}\right)^{\frac{2}{3} \hbar^{2}}}{5 m_{\mathrm{e}} m_{\mathrm{H}}^{\frac{5}{3}}}\left(1+\frac{\alpha}{\eta}\right) \frac{\rho^{\frac{5}{3}}}{\mu_{\mathrm{e}}^{\frac{5}{3}}},
$$

and so one can see that the equation of state is polytropic with index $n=3 / 2$ and polytropic constant

$$
K=\frac{\left(3 \pi^{2}\right)^{\frac{2}{3}} \hbar^{2}}{5 m_{\mathrm{e}} m_{\mathrm{H}}^{\frac{5}{3}} \mu_{\mathrm{e}}^{\frac{5}{3}}}\left(1+\frac{\alpha}{\eta}\right) .
$$

Equation (33) can then be used in Eq. (20) to find the stellar radius

$$
R=\frac{\left(3 \pi^{2}\right)^{\frac{2}{3}}}{5 G m_{\mathrm{e}} m_{\mathrm{H}}^{\frac{5}{3}} \mu_{\mathrm{e}}^{\frac{5}{3}}} \gamma_{3 / 2} M^{-\frac{1}{3}}\left(1+\frac{\alpha}{\eta}\right),
$$

which can be substituted into Eq. (21) to find the core density 


$$
\rho_{\mathrm{c}}=\frac{125 G^{3} m_{\mathrm{e}}^{3} m_{\mathrm{H}}^{5} \mu_{\mathrm{e}}^{5}}{12 \pi^{5} \hbar^{6}} \frac{\delta_{3 / 2}}{\gamma_{3 / 2}^{3}} M^{2}\left(1+\frac{\alpha}{\eta}\right)^{-3} .
$$

The core temperature can be found using Eq. (26),

$$
T_{\mathrm{c}}=\frac{25 G^{2} m_{\mathrm{e}} m_{\mathrm{H}}^{\frac{8}{3}} \mu_{\mathrm{e}}^{\frac{8}{3}}}{2^{\frac{7}{3}} \pi^{2} k_{\mathrm{B}} \hbar^{2}} \frac{\delta_{3 / 2}^{\frac{2}{3}}}{\gamma_{3 / 2}^{2}} \frac{\eta}{(\alpha+\eta)^{2}} M^{\frac{4}{3}} .
$$

In order to find the luminosity $L_{\mathrm{HB}}$ from hydrogen burning, we must integrate the energy generation rate (22) over the volume of the star. Since $\eta$ is constant, we have $T / T_{\mathrm{c}}=$ $\left(\rho / \rho_{\mathrm{c}}\right)^{\frac{2}{3}}$ from Eq. (26), which implies

$$
L_{\mathrm{HB}}=4 \pi r_{\mathrm{c}}^{3} \rho_{\mathrm{c}} \epsilon_{\mathrm{c}} \int_{0}^{\xi_{R}} \xi^{2} \theta^{n\left(u+\frac{2}{3} s\right)} \mathrm{d} \xi .
$$

Using Eq. (15), we have

$\theta(\xi) \approx 1-\left(1+\frac{3 \Upsilon}{2}\right) \frac{\xi^{2}}{6} \approx \exp \left[-\left(1+\frac{3 \Upsilon}{2} \frac{\xi^{2}}{6}\right)\right]$.

One can then perform the integral (37) and use Eq. (19) to find $^{9}$

$$
L_{\mathrm{HB}}=\frac{3 \sqrt{3 \pi}}{\sqrt{2} \omega_{3 / 2}\left[\left(1+\frac{3 \Upsilon}{2}\right)\left(\frac{3}{2} u+s\right)\right]^{\frac{3}{2}}} \epsilon_{\mathrm{c}} M .
$$

Using $u=2.28, s=6.31$, and Eqs. (23), (35), and (36) and inserting the numerical values for the constants, one finds

$\frac{L_{\mathrm{HB}}}{5.2 \times 10^{6} L_{\odot}}=\frac{\delta_{3 / 2}^{5.487}}{\omega_{3 / 2} \gamma_{3 / 2}^{16.46}\left(1+\frac{3 \Upsilon}{2}\right)^{\frac{3}{2}}} M_{-1}^{11.973} \frac{\eta^{10.15}}{(\eta+\alpha)^{16.46}}$,

where $M_{-1}=M / 0.1 M_{\odot}$.

Our next task is to compute the luminosity at the photosphere. ${ }^{10}$ This is the radius where the optical depth, defined as

$$
\tau(r)=\int_{r}^{\infty} \kappa_{\mathrm{R}} \rho \mathrm{d} r
$$

where $\kappa_{\mathrm{R}}$ is the Rosseland mean opacity, is equal to $2 / 3$. It is the photosphere that observations of quantities such as

\footnotetext{
${ }^{9}$ One may worry that the approximation (38) is not accurate since it extends the domain of integration to $\infty$ and is valid only to $\mathcal{O}\left(\xi^{2}\right)$. It is straightforward to verify that the difference between the integral and our approximation is always smaller than $10^{-2}$ over the range $0 \leq \Upsilon \leq 0.05$, which is the range we will investigate below. Physically, the energy generated through nuclear burning is dominated by the central region of the star since the density and temperature fall off quickly, and so the contribution to $L_{\mathrm{HB}}$ comes mainly from the region $\xi \ll 1$.

${ }^{10}$ We denote photosphere quantities with the subscript e.
}

the luminosity and temperature probe and not the stellar surface. The photosphere typically lies very close to the stellar radius, and so the fluid is in the gas phase rather than the metallic one. In GR, one finds the pressure at the optical depth by treating the surface gravity

$$
g \equiv \frac{G M(r)}{r^{2}} \approx \frac{G M(R)}{R^{2}}
$$

as a constant throughout the photosphere and integrating the hydrostatic equilibrium equation. ${ }^{11}$ In alternative theories, we have the additional complication coming from the additional term in Eq. (8). We will deal with this using approximations consistent with those made in GR. In particular, setting $g$ to be constant implies $\mathrm{d}\left(M / r^{2}\right) / \mathrm{d} r=0$, and so we have

$$
\frac{\mathrm{d} M}{\mathrm{~d} r}=2 \frac{M}{r},
$$

which can be differentiated again to find

$$
\frac{\mathrm{d}^{2} M}{\mathrm{~d} r^{2}}=2 \frac{M}{r^{2}}
$$

Equation (8) is then

$$
\frac{\mathrm{d} P_{\mathrm{e}}}{\mathrm{d} r}=-g \rho\left(1+\frac{\Upsilon}{2}\right)
$$

which can be integrated from the photosphere to find

$$
P_{\mathrm{e}}=\frac{2}{3 \kappa_{\mathrm{R}}}\left(1+\frac{\Upsilon}{2}\right) g
$$

where Eq. (41) has been used. Note that modified gravity is then partly degenerate with reducing the opacity. ${ }^{12}$ Indeed, one can define an effective opacity

$$
\kappa_{\mathrm{R}}^{\mathrm{eff}}=\frac{\kappa_{\mathrm{R}}}{\left(1+\frac{\Upsilon}{2}\right)},
$$

which brings all of the equations describing the properties of the photosphere into the same form as GR. Using the ideal gas law, Eq. (46) becomes

$$
\frac{\rho k_{\mathrm{B}} T}{\mu m_{\mathrm{H}}}=\frac{2}{3 \kappa_{\mathrm{R}}}\left(1+\frac{\Upsilon}{2}\right) g .
$$

Unfortunately, this is all the analytic progress we can make with the stellar structure equations alone. In order to

\footnotetext{
${ }^{11}$ This is justified because the distance from the photosphere to the surface is small compared with the stellar radius.

${ }^{12}$ We use the term partly because there are other places where modified gravity has effects that cannot be compensated by changing the opacity.
} 
calculate the effective temperature, we need to know more about the metallic phase transition. To this, we use the approximate analytic fits to the specific entropy $\left(s=S m_{\mathrm{H}} / k_{\mathrm{B}}\right)$ in the gas phase

$$
s_{\text {gas }}=-1.594 \ln \eta+12.43,
$$

taken from [43], and the metallic phase

$$
s_{\text {metallic }}=1.032 \ln \left(\frac{T}{\rho^{0.42}}\right)-2.438,
$$

taken from [44]. Here, $T$ is measured in $\mathrm{K}$ and $\rho$ in $\mathrm{g} / \mathrm{cm}^{3}$. The photosphere is the radius where $s_{\text {gas }}=s$ metallic, and so we have

$$
T_{\mathrm{e}}=1.8 \times 10^{6} \frac{\rho_{\mathrm{e}}^{0.42}}{\eta^{1.545}} \mathrm{~K}
$$

Using Eq. (20), the surface gravity is

$$
\begin{aligned}
g & =\frac{25 G^{3} m_{\mathrm{e}}^{2} m_{\mathrm{H}}^{\frac{10}{3}} \mu_{\mathrm{e}}^{\frac{10}{3}}}{\left(81 \pi^{8}\right)^{\frac{1}{3}} \hbar^{4}} \frac{M^{\frac{5}{3}}}{\gamma_{3 / 2}^{2}}\left(1+\frac{\alpha}{\eta}\right)^{-2} \\
& =\frac{3.15 \times 10^{6}}{\gamma_{3 / 2}^{2}}\left(\frac{M}{0.01 M_{\odot}}\right)^{\frac{5}{3}}\left(1+\frac{\alpha}{\eta}\right)^{-2} \mathrm{~cm} / \mathrm{s}^{2} .
\end{aligned}
$$

Using this and the polytropic relation (33) in Eq. (48) we find

$$
\frac{\rho_{\mathrm{e}}}{\mathrm{g} / \mathrm{cm}^{3}}=5 \times 10^{-5} M_{-1}^{1.17}\left[\frac{\left(1+\frac{\Upsilon}{2}\right)}{\kappa_{-2}}\right]^{0.7} \frac{\eta^{1.09}}{\gamma_{3 / 2}^{1.41}}\left(1+\frac{\alpha}{\eta}\right)^{-1.41},
$$

where $\kappa_{-2}=\kappa / 10^{-2} \mathrm{~cm}^{2} / \mathrm{g}$. Using this in Eq. (51) we find the effective temperature

$$
\frac{T_{\mathrm{e}}}{\mathrm{K}}=2.9 \times 10^{4} \frac{M_{-1}^{0.49}}{\gamma_{3 / 2}^{0.59} \eta^{1.09}}\left[\frac{\left(1+\frac{\Upsilon}{2}\right)}{\kappa_{\mathrm{R}}}\right]^{0.296}\left(1+\frac{\alpha}{\eta}\right)^{-0.59} .
$$

The stellar luminosity $L_{\mathrm{e}}$ is then found by inserting Eqs. (20) and (55) into the formula $L_{\mathrm{e}}=4 \pi R^{2} \sigma T_{\mathrm{e}}^{4}$ to find

$$
L_{\mathrm{e}}=2.65 L_{\odot} \frac{M_{-1}^{1.305}}{\gamma_{3 / 2}^{2.366} \eta^{4.351}}\left[\frac{\left(1+\frac{\Upsilon}{2}\right)}{\kappa_{\mathrm{R}}}\right]^{1.183}\left(1+\frac{\alpha}{\eta}\right)^{-0.366} .
$$

The star can burn hydrogen stably when $L_{\mathrm{HB}}=L_{\mathrm{e}}$, and so equating (37) and (56) one has
$3.76 M_{-1}=\left[\frac{\left(1+\frac{\Upsilon}{2}\right)}{\kappa_{\mathrm{R}}}\right]^{0.11}\left(1+\frac{3 \Upsilon}{2}\right)^{0.14} \frac{\gamma_{3 / 2}^{1.32} \omega_{3 / 2}^{0.09}}{\delta_{3 / 2}^{0.51}} I(\eta)$,

with

$$
I(\eta) \equiv \frac{(\alpha+\eta)^{1.509}}{\eta^{1.325}}
$$

From here on we set $\kappa_{\mathrm{R}}=10^{-2} \mathrm{~cm}^{2} / \mathrm{g}$, which is typical for high-mass brown dwarfs. The stellar composition does not vary between different stars by large amounts, and since Eq. (57) is a weak function of the opacity, the results we will obtain are largely independent of this choice. The weak opacity dependence is one of the reasons dwarf stars are excellent probes of modified gravity. Importantly, the function $I(\eta)$ has a minimum value of 2.34 when $\eta=34.7$. This means that if $M$ is too low, there is no consistent solution to Eq. (57), and hence there is a minimum mass for hydrogen burning. In GR, one has $\gamma_{3 / 2}=2.357, \delta_{3 / 2}=5.991$, and $\omega_{3 / 2}=2.714$, which gives $M_{\mathrm{MMHB}}^{\mathrm{GR}} \approx 0.08 M_{\odot}$. Remarkably, this simple analytic model makes a prediction that is very close to the results of more detailed simulations $[45,46]$, which predict a value of $0.075 M_{\odot}$. In Fig. 2 we plot the MMHB predicted by our alternative theory of gravity as a function of $\Upsilon$; one can see that the MMHB is very sensitive to its value. Large values of $\Upsilon>00^{13}$ raise the MMHB above the GR prediction. This is because the reduced gravity ensures that stars of fixed mass have cores that are cooler and less dense because less nuclear burning is needed to provide the pressure gradient that prevents gravitational collapse. Note that, according to Eq. (56), there is a small effect on the photospheric properties due to modified gravity. These are largely subdominant because they are degenerate with changing the opacity, which effects the MMHB as $\kappa_{\mathrm{R}}^{0.11}$. Most of the deviations from GR are due to changes in the core conditions. Note from Eq. (22) that the rate of hydrogen burning is a strong function of the core temperature and pressure, even stronger than the rate for PP chain burning in main-sequence stars. ${ }^{14}$ This is one reason why dwarf stars are more sensitive probes of alternative gravity theories.

There have been several observations of low mass red dwarf stars in the Milky Way with masses in the range $0.08-0.1 M_{\odot}$ [47-51]. Clearly, large values of $\Upsilon$ are in tension with these observations since they predict that these objects would be brown dwarfs. The lowest mass observed M-dwarf is Gl $866 \mathrm{C}$ [52]; its measured mass is $0.0930 \pm 0.0008 M_{\odot}$. Using Eq. (57), consistency with

\footnotetext{
${ }^{13}$ As discussed above, we will not consider the case $\Upsilon<0$ here because this lowers the MMHB and is hence always consistent with observations of low mass M-dwarfs.

${ }^{14}$ The energy generation on the PP chains is $\epsilon_{\mathrm{PP}} \propto \rho T^{4}$.
} 
this measurement is achieved only when $\Upsilon \lesssim 0.02665$; values larger than this are excluded. In particular, this places a new constraint on the parameters appearing in the EFT,

$$
\frac{\alpha_{H}^{2}}{\alpha_{H}-\alpha_{T}-\alpha_{B}\left(1+\alpha_{T}\right)} \lesssim 0.0068
$$

We end this section by discussing the possible astrophysical degeneracies that could mimic the effects of modified gravity. These are few. We have already seen that the theories considered here are partly degenerate with changing the opacity [see Eq. (47)]. Since the MMHB is only a weak function of the opacity $\left(\propto \kappa_{\mathrm{R}}^{-0.11}\right)$, any variation has only a minimal effect and cannot mimic the large changes induced by modified gravity. The MMHB is also weakly dependent on the amount of stellar rotation, which was absent in our model. Rotation acts to increase the MMHB [53,54] and so can only compound the effect presented here. In order to make a firm prediction, we had to assume specific values for the composition parameters $\mu_{\mathrm{e}}$ and $\mu$. Brown dwarfs show little chemical evolution over their lifetime (partly because their lifetime is so long), and so these are always $\mathcal{O}(1)$. Therefore, our prediction is robust to variations in their values. There are also the effects of missing physics. Our model ignored the effects of mixing and other transport processes. We also assumed an EOS in order to make the problem analytically tractable. When these effects are included in full numerical models [45], one finds that the MMHB changes by less than $0.01 M_{\odot}$, which is far smaller than the changes due to modified gravity. The missing physics is nongravitational, and so it is reasonable to expect similar discrepancies if one were to use full numerical models including the change to the hydrostatic equilibrium equation. The potential changes are an order of magnitude smaller than the changes due to modified gravity, and so the conclusions here should remain largely unchanged, although one should confirm this numerically.

Finally, one must be certain that the empirical mass determination of low mass stars does not implicitly assume GR. If this is the case, our constraint is not self-consistent. Fortunately, this is not the case. The theories considered here do not exhibit deviations from GR outside astrophysical bodies, and Newtonian physics governs the motion of binary objects. The masses of some of the above referenced stars are determined either by using the eclipsing binary technique or by measuring the motions of their satellites. These methods both rely on Newtonian mechanics and not the object's intrinsic properties. For this reason, the mass determination is independent of whether the theory is GR or scalar tensor. If the stars are not in binaries, or do not have any observable satellites, photometry can be used to measure the mass using the mass-luminosity relation. One can see that Eq. (56) implies that this is sensitive to the theory of gravity. The relation used to infer the photometric mass is empirical and not theoretically determined. Indeed, it is calibrated using observations of low mass stars with known mass found using the eclipsing binary technique [55]. For this reason, masses found using this method are insensitive to the theory of gravity.

\section{CONCLUSIONS}

In this paper, we have pointed out that low mass stellar objects, in particular brown and red dwarf stars, are excellent probes of a new and interesting class of alternative theories of gravity: the beyond Horndeski class. Unlike main- and post-main-sequence stars, there are few astrophysical degeneracies, and these are weak functions of nongravitational physics. Furthermore, we have seen that deviations from the GR predictions are non-negligible over a larger range of $\Upsilon$ than in objects such as main-sequence stars and dark matter haloes. For example, main-sequence stars show negligible deviations when $\Upsilon \lesssim 0.1$; this is not the case for dwarf stars.

Low-mass brown dwarfs have radii that are largely independent of their mass. Here, we have presented a simple analytic model of these objects based on $n=1$ polytropes, which make a unique prediction for the radius in terms of fundamental constants and the solution of the Lane-Emden equation. We found that this is smaller than the GR prediction of $0.1 R_{\odot}$ when $\Upsilon<0$ (stronger gravity) and can be as small as $R=0.039 R_{\odot}$, corresponding to the minimum value of $\Upsilon=-2 / 3$. When $\Upsilon>0$, the strength of gravity is reduced and the radius can be arbitrarily large. These are robust predictions of the theory, and more realistic models predict that the physics missing in our model $^{15}$ cause only small deviations in the radius. This means that the effects of modified gravity are not degenerate with the EOS. We do not claim any firm constraint here due to the sparse data in the mass range where our model applies (see [38] and references therein), but we note that future surveys such as GAIA, which will probe this mass range, could place new and independent constraints on $\Upsilon$ and the parameters appearing in the EFT. In particular, the parameter range $-2 / 3 \leq \Upsilon \leq 0$ is not probed by the MMHB, but makes the unique prediction that the radius plateau lies at radii $0.039<R / R_{\odot}<0.1$.

Turning to higher mass brown dwarfs and the brown-red dwarf transition, the minimum mass for hydrogen burning is an extremely sensitive probe of modified gravity. Hydrogen burning requires sufficiently high core temperatures and densities that only sufficiently heavy stars can achieve. General relativity predicts that the MMHB is $\approx 0.08 M_{\odot}$. Here, we have incorporated the effects of alternative gravity theories into the semianalytic model of [28] to predict the MMHB as a function of $\Upsilon$. We have

\footnotetext{
${ }^{15}$ The $n=1$ polytropic approximation is incomplete, and a more realistic EOS is needed.
} 
found that when $\Upsilon>0$, the MMHB is larger than the GR prediction due to the weakening of gravity requiring larger mass stars to achieve the necessary core conditions. Using the upper bound on the mass of the lightest observed M-dwarf, we were able to place the new constraint $\Upsilon \lesssim 0.02665$, which translates into the bound (59) on the parameters appearing in the effective field theory of dark energy. It is interesting to note that the beyond Horndeski covariant quartic Galileon model, which admits self-accelerating cosmological solutions and is therefore a competitor to $\Lambda \mathrm{CDM}$, predicts $\Upsilon=1 / 3$ and is hence excluded by this constraint.

The parameters appearing in the EFT completely characterize the cosmology of beyond Horndeski theories, a very general class of healthy scalar-tensor extensions of GR, on linear scales. Any noncosmological constraint restricts the possible deviations on these scales and is complementary to current and upcoming surveys that will probe the structure of gravity in this regime.

Finally, we end by discussing the applicability of the new tests we have presented here to other alternative gravity theories. The test required deviations from GR in the Newtonian limit and so cannot be applied to any theories that fit into the parametrized post-Newtonian framework, which predicts the inverse-square law. These include theories such as massless scalars and disformal theories [9,21,56-58]. Theories such as massive scalars and vectors as well as screened theories such as chameleons [59] do predict deviations $[60,61]$. In principle, one could apply the tests here to these theories, although in the latter case a comparison with data would be incredibly difficult because one requires objects in other galaxies. Only theories that preserve the scale-invariant nature of the stellar structure equations will result in a modified Lane-Emden equation; theories that do not have this property, such as massive scalars, would require more advanced techniques to extract the MMHB and radius plateau. One important point to make is that the MMHB is very constraining for theories that predict a weakening of the gravitational force but not for theories that predict an enhancement. In this case, the MMHB is lower than the GR prediction, and so the observation of M-dwarfs with masses compatible with GR do not disagree with these theories. Theories where gravity is weaker than the GR prediction are rare because they typically involve extra degrees of freedom such as scalars that couple directly to matter. Since the fifth force is proportional to the square of this coupling, it is difficult to find repulsive forces. Our bound here was obtained using the MMHB, but ultimately it may prove the case that the radius plateau is the more versatile of the tests we have presented here. With upcoming surveys providing better measurements of the mass-radius relation for low-mass objects, this new test of gravity provides another avenue to constrain other modifications of general relativity.

\section{ACKNOWLEDGMENTS}

I would like to thank Kazuya Koyama for many interesting discussions and a careful reading of the manuscript. I am grateful to Michael Cushing, Trent Dupuy, John Gizis, Artie Hatzes, Derek Homeier, and Adam R. Solomon for several enlightening discussions.
[1] T. Clifton, P. G. Ferreira, A. Padilla, and C. Skordis, Phys. Rep. 513, 1 (2012).

[2] A. Joyce, B. Jain, J. Khoury, and M. Trodden, Phys. Rep. 568, 1 (2015).

[3] P. Chang and L. Hui, Astrophys. J. 732, 25 (2011).

[4] A.-C. Davis, E. A. Lim, J. Sakstein, and D. Shaw, Phys. Rev. D 85, 123006 (2012).

[5] B. Jain, V. Vikram, and J. Sakstein, Astrophys. J. 779, 39 (2013).

[6] P. Brax, A.-C. Davis, and J. Sakstein, Classical Quantum Gravity 31, 225001 (2014).

[7] J. Sakstein, Phys. Rev. D 88, 124013 (2013).

[8] V. Vikram, J. Sakstein, C. Davis, and A. Neil, arXiv: 1407.6044

[9] J. Sakstein, B. Jain, and V. Vikram, Int. J. Mod. Phys. D 23, 1442002 (2014).

[10] J. Sakstein, arXiv:1502.04503.

[11] K. Koyama and J. Sakstein, Phys. Rev. D 91, 124066 (2015).

[12] A. Vainshtein, Phys. Lett. 39B, 393 (1972).
[13] E. Babichev, C. Deffayet, and R. Ziour, J. High Energy Phys. 05 (2009) 098.

[14] R. Kimura, T. Kobayashi, and K. Yamamoto, Phys. Rev. D 85, 024023 (2012).

[15] K. Koyama, G. Niz, and G. Tasinato, Phys. Rev. D 88, 021502 (2013).

[16] T. Kobayashi, Y. Watanabe, and D. Yamauchi, Phys. Rev. D 91, 064013 (2015).

[17] J. Gleyzes, D. Langlois, F. Piazza, and F. Vernizzi, Phys. Rev. Lett. 114, 211101 (2015).

[18] J. Gleyzes, D. Langlois, F. Piazza, and F. Vernizzi, J. Cosmol. Astropart. Phys. 02 (2015) 018.

[19] R. Saito, D. Yamauchi, S. Mizuno, J. Gleyzes, and D. Langlois, J. Cosmol. Astropart. Phys. 06 (2015) 008 .

[20] J. Sakstein, Phys. Rev. Lett. 115, 201101 (2015).

[21] G. Esposito-Farese, AIP Conf. Proc. 736, 35 (2004).

[22] A. Nicolis, R. Rattazzi, and E. Trincherini, Phys. Rev. D 79, 064036 (2009).

[23] J. Khoury, arXiv:1312.2006. 
[24] E. Bellini and I. Sawicki, J. Cosmol. Astropart. Phys. 07 (2014) 050.

[25] M. Zumalacárregui and J. García-Bellido, Phys. Rev. D 89, 064046 (2014).

[26] X. Gao, Phys. Rev. D 90, 081501 (2014).

[27] X. Gao, Phys. Rev. D 90, 104033 (2014).

[28] A. Burrows and J. Liebert, Rev. Mod. Phys. 65, 301 (1993).

[29] R. Kippenhahn and A. Weigert, Stellar Structure and Evolution, Astronomy and Astrophysics Library (Springer, New York, 1990).

[30] D. Saumon, W. B. Hubbard, A. Burrows, T. Guillot, J. I. Lunine, and G. Chabrier, Astrophys. J. 460, 993 (1996).

[31] J. Lissauer and I. de Pater, Fundamental Planetary Science: Physics, Chemistry and Habitability (Cambridge University Press, Cambridge, UK, 2013).

[32] S. Chandrasekhar, An Introduction to the Study of Stellar Structure, Dover Books on Astronomy Series (Dover, New York, 2012).

[33] S. S. Kumar, Institute for Space Studies Technical Report No. X-644-62-78, 1962.

[34] C. Hayashi and T. Nakano, Prog. Theor. Phys. 30, 460 (1963).

[35] A. J. Burgasser and C. H. Blake, Astron. J. 137, 4621 (2009).

[36] H. C. Graboske, H. E. Dewitt, A. S. Grossman, and M. S. Cooper, Astrophys. J. 181, 457 (1973).

[37] W. Fowler, G. Caughlan, and B. Zimmerman, Annu. Rev. Astron. Astrophys. 13, 69 (1975).

[38] G. Chabrier, I. Baraffe, J. Leconte, J. Gallardo, and T. barman, AIP Conf. Proc. 1094, 102 (2009).

[39] A. P. Hatzes and H. Rauer, Astrophys. J. Lett. 810, L25 (2015).

[40] A. Sozzetti, S. Casertano, M. G. Lattanzi, and A. Spagna, in Proceedings of the Conference on Towards Other Earths: DARWIN/TPF and the Search for Extrasolar Terrestrial Planets, April 2003, Heidelberg, Germany, edited by M. Fridlund, T. Henning (ESA Publications Division, Noordwijk, 2003), pp. 605-610.

[41] A. Sozzetti, P. Giacobbe, M. G. Lattanzi, G. Micela, R. Morbidelli, and G. Tinetti, Mon. Not. R. Astron. Soc. 437, 497 (2014).
[42] G. Horedt, Polytropes: Applications in Astrophysics and Related Fields, Astrophysics and Space Science Library (Springer, New York, 2006).

[43] A. Burrows, W. B. Hubbard, and J. I. Lunine, Astrophys. J. 345, 939 (1989).

[44] D. Stevenson, Annu. Rev. Astron. Astrophys. 29, 163 (1991).

[45] S. S. Kumar, Astrophys. J. 137, 1121 (1963).

[46] A. Burrows, M. Marley, W. Hubbard, J. Lunine, T. Guillot, D. Saumon, R. Freedman, D. Sudarsky, and C. Sharp, Astrophys. J. 491, 856 (1997).

[47] D. S. Coppenbarger, T. J. Henry, and W. D. McCarthy, Jr., Astron. J. 107, 1551 (1994).

[48] C. Barbieri, G. De Marchi, A. Nota, G. Corrain, W. Hack, R. Ragazzoni, and D. Macchetto, Astron. Astrophys. 315, 418 (1996).

[49] X. Delfosse, T. Forveille, D. Segransan, J.-L. Beuzit, S. Udry, C. Perrier, and M. Mayor, Astron. Astrophys. 364, 217 (2000).

[50] F. Martinache, J. P. Lloyd, M. J. Ireland, R. S. Yamada, and P. G. Tuthill, Astrophys. J. 661, 496 (2007).

[51] T. Hamilton, Our Neighbor Stars: Including Brown Dwarfs, EBL-Schweitzer (Publish on Demand Global LLC, Houston, Texas, 2012).

[52] D. Segransan, X. Delfosse, T. Forveille, J. L. Beuzit, S. Udry, C. Perrier, and M. Mayor, Astron. Astrophys. 364, 665 (2000).

[53] E. E. Salpeter, Astrophys. J. 393, 258 (1992).

[54] R. Kippenhahn, Astron. Astrophys. 8, 50 (1970).

[55] T. J. Henry and D. W. McCarthy, Jr., Astron. J. 106, 773 (1993).

[56] J. Sakstein, J. Cosmol. Astropart. Phys. 12 (2014) 012.

[57] H. Y. Ip, J. Sakstein, and F. Schmidt, J. Cosmol. Astropart. Phys. 10, (2015) 051.

[58] J. Sakstein and S. Verner, Phys. Rev. D 92, 123005 (2015).

[59] J. Khoury and A. Weltman, Phys. Rev. D 69, 044026 (2004).

[60] C. Will, Theory and Experiment in Gravitational Physics (Cambridge University Press, Cambridge, UK, 1993).

[61] L. Hui, A. Nicolis, and C. Stubbs, Phys. Rev. D 80, 104002 (2009). 